\title{
Using simulation to study solvation in water*
}

\author{
R. M. Lynden-Bell ${ }^{1, \ddagger}$, J. C. Rasaiah ${ }^{2}$, and J. P. Noworyta ${ }^{2}$ \\ ${ }^{1}$ Atomistic Simulation Group, School of Mathematics and Physics, Queen's \\ University, Belfast BT7 $1 \mathrm{NN}$, UK; ${ }^{2}$ Department of Chemistry, University of Maine, \\ Orono, ME 04469, USA
}

\begin{abstract}
Simulations of simple solutes (charged and uncharged spheres) in model water have been performed in order to elucidate aspects of solvation in water at ambient and supercritical states. The variation of solvation entropy as a function of solute charge has been used to investigate hydrophobic and hydrophilic ordering and the structure-making and structurebreaking effects of ions. Simulations with model solvents, which differ from water in certain features, have been used to try to identify the particular properties of water that are associated with these phenomena.
\end{abstract}

\section{INTRODUCTION}

This contribution to the $27^{\text {th }}$ International Conference on Solution Chemistry describes some of our recent work on solvation of ions and small solvents in water, which is a rather unusual molecular liquid. We shall discuss the role that simulation can play in assisting our understanding of solvation at the molecular level. Our particular interest in this paper is the solvation of small atomic ions and small spherical uncharged, hydrophobic solutes in water both at room temperature and at supercritical temperatures.

Water at ambient and at biological conditions is a common and important solvent. As is well known, it is a good solvent for ions and polar molecules (hydrophilic solutes), and an exceptionally poor solvent for nonpolar or hydrophobic solutes $[1,2]$. The phenomenon of hydrophobicity has been discussed for many years before and there are many relevant experimental measurements [3]. In recent years, simulation has been added to the range of tools used to try to understand this phenomenon. In fact, there are a range of phenomena included in the term hydrophobic effect-the low solubility of methane in water compared to methanol or organic liquids; the immiscibility of oil and water; the wetting or non-wetting of surfaces by water; hydrophobic hydration. Here, we shall be concerned with the solvation of small solutes in very dilute solutions.

It is generally understood that hydrophobicity is a property of the water rather than the solute. It is not so much that methane is water-hating but that water is methane-hating [4]. The properties of liquid water depend on the intermolecular potential between water molecules and in particular on the formation of hydrogen bonds. In ice, each water molecule forms four hydrogen bonds (two donated and two accepted), which results in a rather open network structure. In the liquid under ambient conditions, this structure persists to some extent, at least at short range, and it is this that is thought to give water its unique properties. The interaction of ions with water can lead to breaking of this structure, which is replaced by a different ordering of the molecules in the local field of the ion. The balance between the structure-breaking and structure-making tendencies can be seen in the entropy of solvation, which we shall examine in simulations. It has also been proposed that hydrophobic solutes are intrinsically structure-making, that is, they induce local structure around themselves in what has been described as iceberg formation. Simulations have shown that this probably does not occur to any significant extent.

\footnotetext{
*Plenary lecture presented at the $27^{\text {th }}$ International Conference on Solution Chemistry, Vaals, The Netherlands, 26-31 August 2001. Other presentations are published in this issue, pp. XXXX-XXXX.

${ }^{\ddagger}$ Corresponding author
} 
Supercritical water is a fluid above the critical point. The density can be varied continuously over a wide range of values from gas-like to liquid-like. It is commercially important as it can be used as a solvent for reactions (e.g., oxidation of waste) and then removed by reducing the density. It also has the great advantage of being nontoxic. Hydrogen bonds are less important at these high temperatures, and the solvation properties are more normal or organic-like. The fact that the density can be varied widely makes it an interesting medium for theoretical studies of solvation, as the effects of density and temperature changes can be separated.

\section{SIMULATION}

There are a number of types of atomistic simulation available for studies. While quantum simulations are more accurate, they are very expensive computationally and it is only realistically possible to do small simulations (50 molecules or less) for short times (a few ps). This is quite inadequate for exploring the range of configurations that are typical of a solution, and hence classical simulations are appropriate. These can be carried out for hundreds (or even thousands) of molecules for times of a nanosecond or longer.

As the aim is to study microscopic aspects of solvation and the relationship between microscopic and macroscopic properties, it is not necessary to have complete quantitative accuracy (the aim is not to supersede experimental work, but to complement and understand it). However, the model must contain the correct physics. Thus, the model intermolecular potential can be approximate, but must not be too crude. One real advantage of simulations is that there is the possibility of changing the model (or the state point) to inaccessible or unphysical values. In this work, for example, we use ions with charges of a fraction of an electron in order to understand the solvation thermodynamics. One should note that even in classical simulations there are severe limitations of time scale, distance scale, and accessible concentration range.

\section{Intermolecular potentials for water}

The basic physics that must be included in the model are the molecular size, the molecular shape, and the intermolecular interactions. These are usually described by an intermolecular site-site potential with several sites on each molecule. The inter-site interactions include repulsion at short distances which describes the shape of the molecule; attractive dispersion (van der Waals') interactions at intermediate range; and electrostatic interactions. The latter may be attractive or repulsive, are both short and long range, and are particularly important in determining the local arrangement of molecules in the liquid. There are other terms that contribute to intermolecular forces, such as partial covalency and chargetransfer, but, provided that no chemical bonds exist between molecules, it is sufficient to include repulsion, dispersion, and electrostatic terms even for hydrogen-bonded liquids.

The work described here used the SPC/E model for water [5]. This is one of a number of models with fixed charges on a few sites and a Lennard-Jones center on the oxygen atom. In this particular model there are 3 sites $(\mathrm{O}$ and two $\mathrm{H})$ with a bond length of $1 \AA$ and a tetrahedral bond angle. As the molecule is neutral and symmetric, the electrostatics is described by a single parameter (the charge on the oxygen atom, say). Two parameters describe the Lennard-Jones interaction and two the geometry. The molecular size is determined by the value of the Lennard-Jones sigma parameter, while the directionalities of the intermolecular interactions including the hydrogen bonds are determined by the partial charges and the positions of the $\mathrm{H}$ sites relative to the oxygen. This model is remarkably successful for modeling liquid water under ambient conditions and is reasonably successful under other conditions. We may conclude that it contains the essential physics of the intermolecular potential, although we would not expect it to be quantitatively accurate. One missing ingredient is the effect of polarizability. In real liquids, the instantaneous electron distribution in each molecule responds to the instan- 
taneous environment, while in this model the electrostatic properties of each molecule are fixed. The dipole moment at $2.35 \mathrm{D}$ is higher than in the gas phase $(1.85 \mathrm{D})$, representing the average effect of the polarization due to the environment, but does not fluctuate. This large enhancement is supported by $a b$ initio calculations which, when analyzed in different ways, give values of $2.45 \mathrm{D}$ [6] or $2.95 \mathrm{D}$ [7] in the liquid state, however, the same calculations show significant fluctuations.

While the use of this model is reasonable for ambient conditions, we have also used it in the supercritical region where the effects of fluctuations may be more important and the average polarization less. There are polarizable models [8,9] that are used in this region, but the aim of our work is to try to isolate effects of temperature and density on solvation using the simplest realistic model available. An important point is that the critical point and static dielectric properties of SPC/E water are not too different from real water $[10,11]$.

Our calculations [12-18] have been carried out using standard methods of molecular dynamics [19], with some extensions in the runs with variable size or charge [12]. It is important to treat the longrange part of the electrostatics in a consistent way. We have used both the reaction field method and the Ewald summation method. The reader is referred to the original papers for more details of the simulations.

\section{SOLVATION OF IONS AND UNCHARGED SPHERES AT AMBIENT CONDITIONS}

We have investigated the solvation of spherical solutes in very dilute solutions, that is, with one solute molecule per simulation box. The solvation thermodynamics is defined by considering a box of volume $V$ containing $N$ water molecules (this is the periodically repeated unit in the simulation). The solvation energy, free energy, entropy, etc. are defined as the difference in these quantities for the box containing the water molecules and the solute molecule and two separate isolated boxes of volume $V$, one with the water molecules and one with the solute molecule. One can also consider this as the difference between the thermodynamic properties of the box with the solute molecule and the water molecules when they interact and when they do not interact. As the volume is held constant, the natural thermodynamic variables are the Helmholz free energy, $A$, and the internal energy $U$, rather than the Gibbs free energy and the enthalpy. The solvation free energy is related to the Ostwald solubility $L_{\text {ost }}$ of a gas by $A_{\text {solv }}=-k T \ln L_{\text {ost }}$ and is equal to the chemical potential of the solute. It is possible to measure $A_{\text {solv }}$ and $U_{\text {solv }}$ from simulations and hence to determine the solvation entropy $S_{\text {solv }}$ from their difference. It should be noted that $S_{\text {solv }}$ is the partial derivative of the chemical potential with respect to temperature at constant volume and differs from the partial molar entropy of the solute, which is the partial derivative of the chemical potential with respect to temperature at constant pressure. The difference is negligible at ambient conditions, but may be significant in the supercritical region.

The solutes considered are simple Lennard-Jones spheres with a single charge. The solvation thermodynamics depends on just three parameters, the oxygen charge, $q$, the size $\sigma$, and the well depth $\varepsilon$ of the Lennard-Jones interaction between the solute molecule and the water oxygen. We have kept the Lennard-Jones well depth constant and equal to the value [20] recommended for a number of atomic ions and determined the free energy as a function of charge and size. This is done by a sequence of simulations:

- $\quad$ For zero charge and small values of solute size, the solvation free energy was found by the Widom particle insertion method. This gives an absolute value for the free energy.

- For larger sizes, the variation of free energy with size was determined by thermodynamic integration of the average derivative of the energy with respect to the value of $\sigma$. This was done in an extended ensemble simulation in which the size was allowed to vary.

- With a fixed value of $\sigma$ (that for a $\mathrm{Na}^{+}$ion) the variation of the solvation free energy with charge was found by similar methods.

- $\quad$ This was repeated for other values of the solute size. 
Figure 1 shows the variation of the solvation free energies with charge for solutes of two different sizes. The first point to note is that the chemical potential decreases (the solute is more stabilized by solvation) as the magnitude of the charge increases. This is primarily due to electrostatic interactions. and is what would be expected from the Born model. It can be partly explained by the lowering of the free energy in solution compared to the gas phase by the polarization of the medium by the charge. This is a particularly large effect due to the high dielectric constant of water. However there are features that are peculiar to the molecular structure of water, in particular the considerable asymmetry between positive and negative ions. Negative ions act as hydrogen bond acceptors and so have a greater and asymmetrical interaction with the nearest water molecules than the positive ions do. In the latter case, the water molecules align with their dipole moments pointing (on average) along the radial directions from the ion. A molecular description of water is necessary to describe this asymmetry as continuum models, such as the Born model, predict a symmetric quadratic dependence of the free energy on the charge.

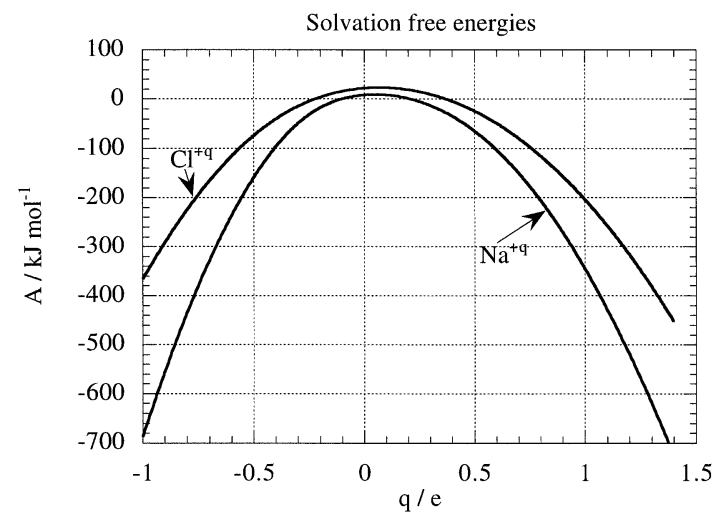

Fig. 1 Variation of the solvation free energies (chemical potentials) of spheres the size of sodium and chloride ions with ion charge at room temperature.

\section{Solvation entropies}

Figure 2 shows the solvation entropies for solutes the size of a sodium and a chloride ion, respectively. Further examples are given in our paper [12]. While the solvation free energy depends on both shortand long-range interactions, the solvation entropy is a more local effect, and is more dependent on the molecular nature of the solvent. The unusual double maximum in these curves is an interesting feature of the curves in Fig. 2. At high values of the magnitude of the charge, the solvation entropy becomes more negative as the solvent molecules become aligned in the electric field of the ion. One anticipates this latter "structure-making effect" in any solvent formed from polar molecules. The double maximum, on the other hand, is less common. The increase in solvation entropy with charge at small magnitudes of the charge is the result of structure-breaking by the ion, which depends, of course, on the solvent having structure to be broken. The double maximum occurs when the structure-making balances the structure-breaking. This is an old concept, which can be demonstrated unambiguously in these simulations as the charge can be varied continuously and independently of other parameters in simulations.

The extent and pattern of structure-breaking depends on the size of the solute. For larger solutes, the depth of the minimum and the separation between the two maxima increases, showing that the structure-making tendencies take longer to swamp the structure-breaking tendencies. We note that again there is considerable asymmetry between positive and negative ions of the same size. The positive ion $\mathrm{Na}^{+}$is a net structure maker in the sense that $q=1$ lies well outside the two double maxima. Structurebreaking and structure-making are about equal for an ion the size of $\mathrm{Cs}^{+}$while larger ions such as $\mathrm{Rb}^{+}$ 


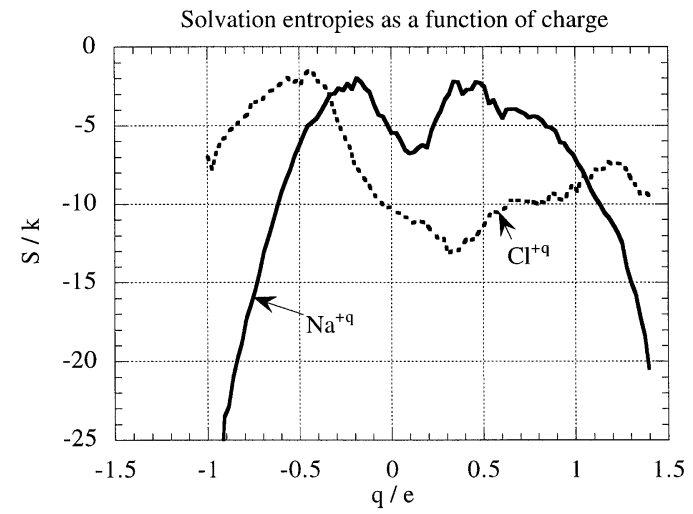

Fig. 2 Variation of the solvation entropies of spheres the size of sodium and chloride ions with ion charge at room temperature.

lie on the inside of the maximum and so can be described as structure-breakers. The negative ions are all net structure-makers according to this definition with $\mathrm{I}^{-}$lying just beyond the maximum for a negatively charged solute. However, there are other possible definitions of structure-breaking and structuremaking, and it has been more common to base these terms on the absolute values of the solvation entropies.

Indeed another way of thinking about these curves is to concentrate on the difference between the observations and those expected for a simple polar solvent. From this point of view, it is the region of low charge that is anomalous and we can describe the abnormally low solvation entropy of uncharged solutes to hydrophobic ordering. The low entropy associated with the solution of hydrophobic solutes has also been known for many years from experiments and was ascribed to a local freezing of the water induced by the solute, sometimes known as the iceberg effect. However, the word "freezing" implies a decrease in enthalpy as well as entropy, which is not found. One can now ask the question "What is special about water which gives rise to all the phenomena described as hydrophobic?" A supplementary question is whether this double maximum can be used as a signature of the hydrophobic effect.

From the measurements of the solvation thermodynamics of uncharged spheres as a function of size (see operations 1 and 2 above), we find that the energy of solvation is essentially zero and the free energy of solvation is determined by the entropy. The fact that the energy is very small shows that the entropy of solvation is determined by the probability of finding a cavity in the liquid that is large enough to insert the sphere. Thus, it depends both on the free volume in the liquid and its distribution. It is not surprising that the free energy of solvation is positive and increases with solute size, or alternatively that the entropy of solvation is negative and becomes more negative as the solute size increases.

One important factor that contributes to the large negative entropy of solvation is simply that the water molecules are smaller than the molecules of most organic liquids. For the same fraction of free volume, the size of the voids scales with the size of the solvent molecules and much of the abnormally low entropy of solvation of hydrophobic solutes can simply be attributed to the small size of water molecules. This is only indirectly a property of the water molecule in that, if it did not have hydrogen bonds, it would not be a liquid under ambient conditions. However, this explanation is insufficient to account for the more subtle effects of hydrophobicity such as the double maximum in the entropy versus charge curves. The existence of a network structure might be expected to be important in this context. It has been shown [22] that the distribution of cavities in the neat liquid is sharpened by the network structure, lowering the solvation entropy for larger solutes.

Another contribution to the solvation entropy of a nonpolar solute is from the formation of a hydrogen-bonded cage enclosing the solute. This is confirmed in computer simulations by visual obser- 
vation of equilibrium configurations of charged solutes in water at room temperature. The residence times of water molecules in the primary hydration shells of discharged sodium $\mathrm{Na}^{0}$, chloride $\mathrm{Cl}^{0}$, and iodide $\mathrm{I}^{0}$ in SPC/E water are approximately 9,18 , and 24 picoseconds respectively at $298 \mathrm{~K}$. The lifetimes of these cages are relatively long, and they increase with the size of the nopolar solute. The cage begins to break down when the solute is charged, and the solvation entropy rises to a maximum. Further increases in the charge lower the entropy as the solvent molecules are ordered by the electric field of the solute. This alignment is determined not only by the magnitude, but also by the sign of the electric charge. The residence times of water in the primary hydration shells of $\mathrm{Na}^{+}, \mathrm{Cl}^{-}$, and $\mathrm{I}^{-}$ions in SPC/E water are approximately 20,13 , and 9 picoseconds at room temperature. They decrease with the size of the solute, which is the opposite of what is observed for the uncharged solutes.

Charging a solute in aqueous solution at room temperature progressively changes the entropy from typically hydrophobic to hydrophilic solvation. The double maximum and asymmetry in the solvation entropy as a function of charge are reflections of the solvent response to the solute, determined by the charge distribution and hydrogen bonding of the water molecules, and the magnitude and sign of the solute charge. In contrast to this, the continuum Born model predicts a symmetric quadratic dependence of the entropy of solvation on the solute charge, assuming a negative temperature dependence of the static dielectric constant of the solvent that occurs in water. The characteristic signature of hydrophobic solvation is less prominent or even absent at supercritical water, which is discussed in the last section.

\section{Simulations of "not-water"}

In order to investigate the importance of the molecular properties of water further, Bergman and Lynden-Bell [23] performed simulations of a number of highly distorted water models and investigated the solvation properties of these liquids. The idea was to keep the same size of solvent molecule, but to vary the intermolecular interactions. Two of the distorted molecules had bond angles reduced to $90^{\circ}$ and $60^{\circ}$ respectively, but had the same value for the dipole moment so that the long-range interactions were the same. The third model has reduced charges, but unchanged geometry. Simulations of the neat liquids showed that the network structure changed considerably as the geometry was distorted. Indeed, the $60^{\circ}$ water showed chains of molecules rather than any rings or remnants of a three-dimensional structure. The intermolecular interactions were still strong. Reducing the charges merely reduced the extent of structure in the first shell, making the liquid more like an organic liquid. The number density was the same for all the liquids. In order to compare the same state points, the Lennard-Jones $\sigma$ was adjusted until the pressure was near zero.

It was pointed out earlier that the solvation thermodynamics of uncharged spheres was primarily determined by the probability distribution of cavities of different sizes in the liquid and that this was a particularly sharp function for water as compared to organic liquids. This distribution was found to be very similar for normal water and $90^{\circ}$ water, and only slightly broader for $60^{\circ}$ water. In the reducedcharge water, however, the distribution was significantly broader and more like that in an organic liquid. The result of this distribution of cavities is that the free energies of solvation for an uncharged sphere of a given size are only slightly lower for $90^{\circ}$ water and $60^{\circ}$ water than for SPC/E water and significantly lower for the reduced-charge water. Thus, changing the geometry, although it has a profound effect on the network structure, does not seem to greatly affect the solvation properties of the liquid toward hydrophobic solutes.

The entropy versus charge curves that are shown in Fig. 3 reinforced this unexpected conclusion. Although the hydrophobic minimum is somewhat less pronounced for the distorted waters than for normal water, it is clearly present. We conclude that the existence of a three-dimensional network is not essential for hydrophobic-like phenomena. 


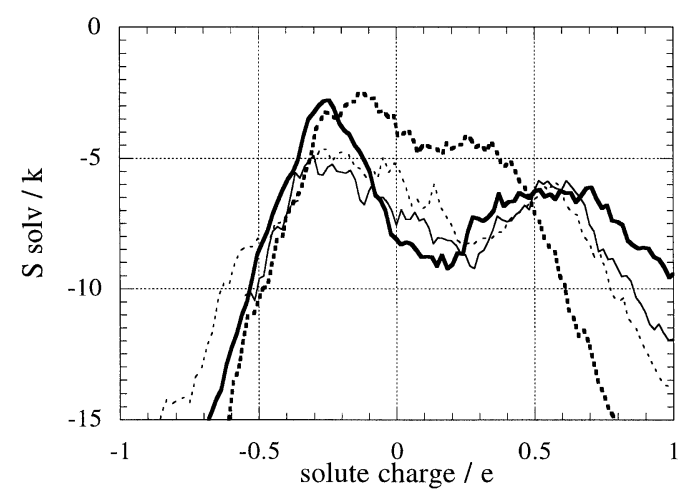

Fig. 3 Solvation entropy, $S_{\text {solv }}$, as a function of charge for spheres the size of a sodium ion in SPC/E water (bold line), $90^{\circ}$ water (thin line), $60^{\circ}$ water (thin dotted line), and reduced-charge water (bold dotted line).

\section{Simulations of supercritical water}

It is difficult to do simulations in the vicinity of the critical point as the length scales and time scales of density fluctuations get very large. However, at $683 \mathrm{~K}$, the temperature is nearly $40 \mathrm{~K}$ above the critical temperature of both the model $\left(T_{\mathrm{c}}=647 \mathrm{~K}\right)$ and real water $\left(T_{\mathrm{c}}=640 \mathrm{~K}\right)$ and the critical fluctuations are no longer an issue. We have performed simulations $[16,17,24]$ at three solvent densities $0.20,0.35$, and 1.00 times the density of ambient temperature $\left(\rho=0.997 \mathrm{~g} \mathrm{~cm}^{-3}\right.$ or $\left.997 \mathrm{Kg} \mathrm{m}^{-3}\right)$. Comparing the high-density state point with the room-temperature state point shows the effects of temperature at constant density, while comparing the three high-temperature state points shows the effects of changing density at constant temperature. Considering first the changes in the structure of the neat liquid at constant density (Fig. 4) one finds that the most obvious effect of temperature on the oxygen-oxygen radial distribution function is in the region of the second peak rather than the first peak. In atomic liquids, the second peak in the radial distribution function is at twice the distance from the origin than the first peak, but in liquid water under ambient conditions the second peak is considerably closer. This is a signature of the tetrahedral coordination and network structure in liquid water. At $683 \mathrm{~K}$ and high density, the second peak is much less pronounced, and more importantly is at twice the distance of the first peak. This is one of several indications that the local coordination is no longer dominated by hydrogen bonding and that the network has broken down. As the density is reduced at $683 \mathrm{~K}$, the first peak in the radial distribution function becomes stronger, showing that there is some tendency for the water molecules to cluster.

These changes lead us to expect that the solvation properties of supercritical water may be very different from those of ambient water. Indeed, this is so; experimentally it is found that at densities of around 0.35 of the ambient density sodium chloride is no longer soluble and alkanes become miscible with water. Simulations [18] have also shown that a solution of sodium and chloride ions in water soon crystallizes into small clusters of solid sodium chloride.

Figure 5 shows the entropies of solvation of small uncharged spheres as a function of radius. The energies of solvation are very small, and the solvation free energies are dominated by the entropies of solvation. As the temperature is raised at constant density there is no significant change in the entropies of solvation, which become more negative with increasing size. If, however, the density is lowered, the entropy of solvation is much less significant, although it remains negative. There are two points to make in this context; first, that the variation with density can be explained in terms of the increased free volume in the less-dense liquid and the consequent occurrence of larger cavities, and secondly that, as in the not-water models, the breakdown of the liquid network has less effect on the solvation of hydrophobic solutes than might be expected. 


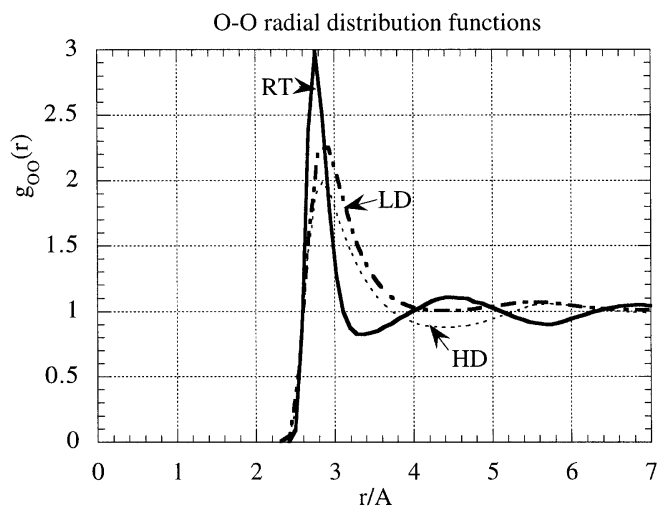

Fig. 4 Radial distribution functions of SPC/E water at $298 \mathrm{~K}, \rho=0.997 \mathrm{~g} \mathrm{~cm}^{-3}$ (solid line) $683 \mathrm{~K}, \rho=0.997 \mathrm{~g} \mathrm{~cm}^{-3}$ (dashed line) and $683 \mathrm{~K}$ and $\rho=0.35 \mathrm{~g} \mathrm{~cm}^{-3}$ (dot-dashed line).

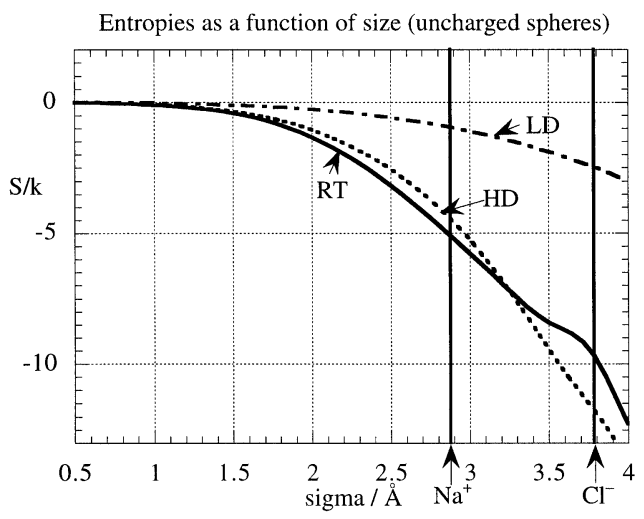

Fig. 5 Solvation entropies, $S_{\text {solv }}$, of uncharged spheres as a function of radius at a number of state points: $298 \mathrm{~K}$, $\rho=0.997 \mathrm{~g} \mathrm{~cm}^{-3}$ (solid line) $683 \mathrm{~K}, \rho=0.997 \mathrm{~g} \mathrm{~cm}^{-3}$ (dashed line) and $683 \mathrm{~K}$ and $\rho=0.35 \mathrm{~g} \mathrm{~cm}^{-3}$.

Figure 6 shows the solvation entropy of spheres the size of a sodium ion with a variable charge. Comparing the two curves at different temperatures and the same density, one sees that the solvation entropy curve is a much flatter function of charge at high temperatures than at low temperatures. This is because the hydrophilic ordering of the water in the field of the ion is less at high temperatures where the thermal motion is greater. However, the low-density high temperature state point shows the opposite trend with a steeper curve than under ambient conditions. Here, although the thermal motion is increased from ambient conditions, the solvent molecules have much greater freedom to move at low densities and as the charge on the ion is increased, they cluster around the ion and the order is reduced. This is shown clearly in Fig. 7.

In these radial distribution functions, which show the ratio of the density at distance $r$ from the ion to the average density, it is clear that a sodium ion is an enormous perturbation on the supercritical water, while the uncharged sphere provides a negligible perturbation. The ion nucleates ordered clusters of water molecules while the uncharged sphere does not. This makes the entropy of solvation of ions in less-dense supercritical water very negative, but this is due to an increase in local density, while the main contribution to the hydrophilic ordering at room temperature is an increase in orientational order of the water molecules in the field of the ion. 


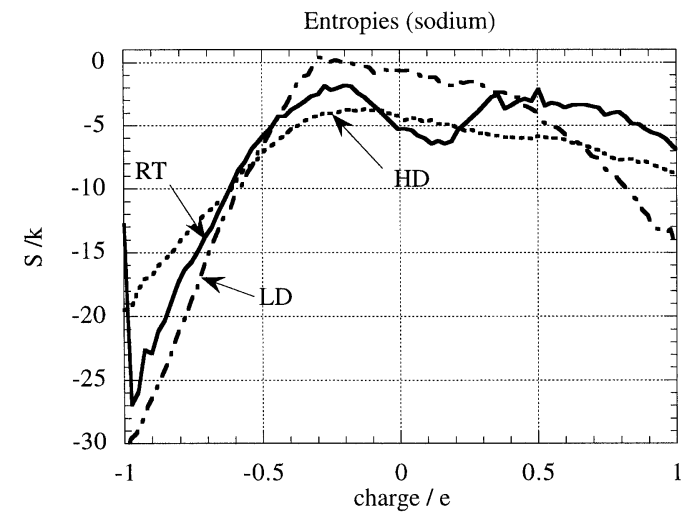

Fig. 6 Solvation entropies, $S_{\text {solv }}$, of charged spheres the size of a sodium ion as a function of charge at a number of state points: $298 \mathrm{~K}, \rho=0.997 \mathrm{~g} \mathrm{~cm}^{-3}$ (solid line) $683 \mathrm{~K}, \rho=0.997 \mathrm{~g} \mathrm{~cm}^{-3}$ (dashed line) and $683 \mathrm{~K}$ and $\rho=0.35 \mathrm{~g} \mathrm{~cm}^{-3}$.

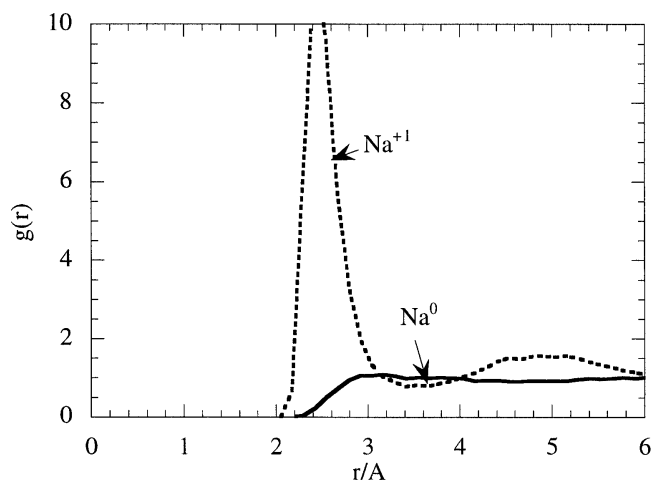

Fig. 7 Radial distribution functions for water oxygen around a sodium ion (dashed line) and around an uncharged sphere the same size at the state point $T=683 \mathrm{~K}$ and $\rho=0.35 \mathrm{~g} \mathrm{~cm}^{-3}$.

The other important change at both the high-temperature state points is the disappearance of the local minimum in the graphs of solvation entropy as a function of charge. We deduce that structuremaking due to electrostatic hydrophilic solvation still occurs at supercritical temperatures, especially at low densities, but structure-breaking is no longer seen. This can be explained as due to the breakdown in the cages around uncharged solutes when the temperature is raised to supercritical values. Figure 8 compares the solute-water distribution functions for discharged sodium $\left(\mathrm{Na}^{0}\right)$ at low and high temperatures. The peaks in the solute-water distribution functions at the high-density state point $(\mathrm{HD}, T=683 \mathrm{~K}$, $r=0.997 \mathrm{~g} \mathrm{~cm}^{-3}$ ) are located at slightly shorter distances than those observed at room temperature (RT, $T=298 \mathrm{~K}$ and $r=0.997 \mathrm{~g} \mathrm{~cm}^{-3}$ ) and are mainly due to solvent packing. The residence times of water in the primary hydration shells of discharged $\mathrm{Na}^{0}, \mathrm{Cl}^{0}$, and $\mathrm{I}^{0}$ at $683 \mathrm{~K}$ and solvent density of $0.997 \mathrm{~g} \mathrm{~cm}^{-3}$ are all of the order of $2-3$ picoseconds in contrast to the much higher values $(9,18$, and 24 picoseconds respectively) at RT, suggesting the absence of cages at high temperatures. The low-density supercritical state (LD, $T=683 \mathrm{~K}$ and $r=0.35 \mathrm{~g} \mathrm{~cm}^{-3}$ ) is characterized by the absence of a hydration shell around the uncharged solute and, in our simulations, no measurable residence time for water in this shell. 


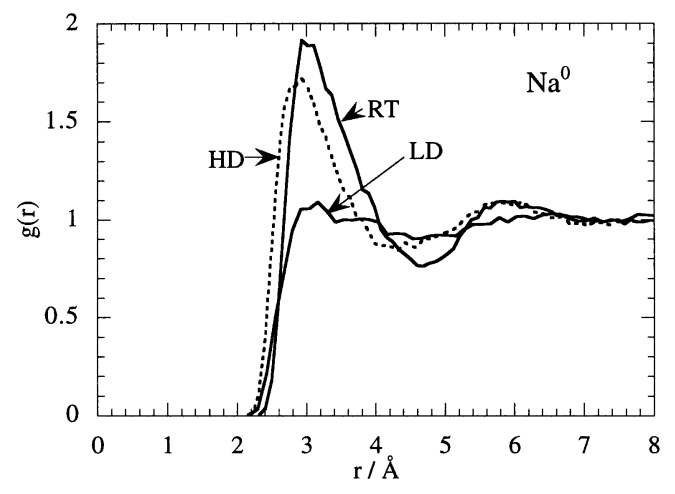

Fig. 8 Radial distribution functions for water around uncharged sodium $\mathrm{Na}^{0}$ at room temperature (RT, $298 \mathrm{~K}$ and $\left.\rho=0.997 \mathrm{~g} \mathrm{~cm}^{-3}\right)$ at a high-density supercritical state $\left(\mathbf{H D}, T=683 \mathrm{~K}, \rho=0.997 \mathrm{~g} \mathrm{~cm}^{-3}\right.$ and low-density supercritical state (LD, $683 \mathrm{~K}$ and $\rho=0.35 \mathrm{~g} \mathrm{~cm}^{-3}$ ).

\section{CONCLUSIONS}

In this study, we have shown how a simple model of water, which has hydrogen-bonding, can be used to study aspects of solvation. Our emphasis has been on studying simple solutes (charged and uncharged spheres of different sizes) in order to try to elucidate and find a microscopic explanation of the thermodynamics of solvation. The entropy of solvation has proved to be a particularly informative property. The abnormally low entropy of solvation associated with hydrophobic solutes has been shown by us and by others [21,22] to be a property of the distribution of cavities in the liquid, which in turn depends on the small size of the solvent molecules. The structure-breaking effect, which leads to an increase of entropy as the charge on the sphere is slowly turned on, is more directly associated with "structure" in the liquid. This leads to a characteristic double maximum in the solvation entropy versus charge curves. Our numerical experiments on solvents ("not-waters") show that this double maximum persists in liquids with strong intermolecular interactions even when the local structure is quite different from that in water. If the relative strength of the hydrogen-bonding compared to thermal energies is decreased either by changing the intermolecular potential (reduced-charge water) or by increasing the temperature, structure-breaking is reduced and the double maximum is no longer seen. In supercritical liquids the density has a large effect on the nature and extent of hydrophilic ordering.

Simulation does not replace experiments but complements them. By altering the intermolecular potential in a controlled way, it has been possible to unravel some of the factors affecting subtle phenomena such as the hydrophobic effect.

\section{ACKNOWLEDGMENTS}

We thank the EPSRC (grants GR/M72401 and GR/L08427), the NSF (grant CHE 9961336), and SIMU for funding associated with this project and the University of Maine for the award of a Libra Visiting Professorship to RMLB.

\section{REFERENCES}

1. F. Franks. Water, A Comprehensive Treatise, Plenum, New York (1973).

2. H. Eisenberg and W. Kauzmann. The Structure and Properties of Water, Oxford University Press, Oxford (1969).

3. A. Ben-Naim. Hydrophobic Interactions, Plenum Press, New York (1980). 
4. A. D. J. Haymet, K. A. Silverstein, K. A. Dill. Farad. Disc. 103, 117 (1996).

5. H. D. C. Berendsen, J. R. Grigera, T. P. Straatsma. J. Phys. Chem. 91, 6269 (1987).

6. L. Delle Site, A. Alavi, R. M. Lynden-Bell. Mol. Phys. 96, 1683 (1999).

7. P. L. Silvestrelli and M. Parrinello. J. Chem. Phys. 111, 3572 (1999).

8. A. A. Chialvo and P. T. Cummings. J. Chem. Phys. 101, 4466 (1994).

9. S. Koneshan, J. C. Rasaiah, L. X. Dang. J. Chem. Phys. 114, 75441 (2001).

10. Y. Guissani and B. J. Guillot. J. Chem. Phys. 98, 8221 (1993).

11. R. Mountain and A. Wallquist. "A collection of results for the SPC/E water model." National Institutes of Standards and Technology Report NISTIR. 5778 (1996).

12. R. M. Lynden-Bell and J. C. Rasaiah. J. Chem. Phys. 107, 1981 (1997).

13. J. C. Rasaiah and R. M. Lynden-Bell. Phil. Trans. Roy. Soc. (London) A (2001) In press.

14. S. Koneshan, S. H. Lee, R. M. Lynden-Bell, J. C. Rasaiah. J. Phys. Chem. B, 102, 4193 (1998).

15. S. Koneshan, S. H. Lee, R. M. Lynden-Bell, J. C. Rasaiah. J. Amer. Chem. Soc. 120, 12041 (1998).

16. J. C. Rasaiah, J. P. Noworyta, S. Koneshan. J. Amer. Chem. Soc. 122, 11182 (2000).

17. J. P. Noworyta, S. Koneshan, J. C. Rasaiah. J. Amer. Chem. Soc. 122, 11194 (2000).

18. S. Koneshan and J. C. Rasaiah. J. Chem. Phys. 113, 8125 (2001).

19. M. P. Allen and D. J. Tildesley. Computer Simulation of Liquids, Oxford University Press, Oxford (1987).

20. D. E. Smith and L. X. Dang. J. Chem. Phys. 100, 3757 (1994).

21. A. Pohorille and L. R. Pratt. J. Amer. Chem. Soc. 112, 5066 (1990).

22. L. R. Pratt and A. Pohorille. Proc. Natl. Acad. Sci. 89, 2995 (1992).

23. D. L. Bergman and R. M. Lynden-Bell. Mol. Phys. 99, 1011 (2001).

24. J. P. Noworyta, R. M. Lynden-Bell, J. C. Rasaiah. In preparation. 